\title{
Assessment of 3D scanners for modeling Pectus Carinatum corrective bar
}

\author{
António H. J. Moreira, ${ }^{1,2,}$, João Gomes Fonseca ${ }^{2, *}$, Pedro L. Rodrigues ${ }^{1,2}$, Jaime C. Fonseca ${ }^{1,5}$, A. C. \\ M. Pinho ${ }^{6}$, Jorge Correia-Pinto ${ }^{2}$, Nuno F. Rodrigues ${ }^{3,4}$, and João L. Vilaça ${ }^{1,2,3}$ \\ ${ }^{I}$ Centro ALGORITMI -School of Engineering, University of Minho, Guimarães, Portugal \\ ${ }^{2}$ ICVS/3B's - PT Government Associate Laboratory, Braga/Guimarães, Portugal \\ ${ }^{3}$ DIGARC -Polytechnic Institute of Cávado and Ave, Barcelos, Portugal \\ ${ }^{4}$ HASLab / INESC TEC -University of Minho, Braga, Portugal \\ ${ }^{5}$ DEI-Industrial Electronics Department, University of Minho, Guimarães, Portugal \\ ${ }^{6}$ DEM-Mechanical Department, University of Minho, Guimarães, Portugal \\ ${ }^{*}$ These authors contributed equally to this work
}

Keywords: $\quad$ 3D scanner, Kinect, FastSCAN, Pectus Carinatum, Surface mesh, 3D reconstruction

Abstract: Pectus Carinatum (PC) is a chest deformity consisting on the anterior protrusion of the sternum and adjacent costal cartilages. Non-operative corrections, such as the orthotic compression brace, require previous information of the patient chest surface, to improve the overall brace fit. This paper focuses on the validation of the Kinect scanner for the modelling of an orthotic compression brace for the correction of Pectus Carinatum. To this extent, a phantom chest wall surface was acquired using two scanner systems Kinect and Polhemus FastSCAN - and compared through CT. The results show a RMS error of $3.25 \mathrm{~mm}$ between the CT data and the surface mesh from the Kinect sensor and $1.5 \mathrm{~mm}$ from the FastSCAN sensor.

\section{INTRODUCTION}

Pectus Carinatum (PC) is a chest deformity characterised by the anterior projection of the sternum and adjacent costal cartilages. The deformity is caused by a disproportionate growth of the costal cartilages, compared to the thoracic skeleton, resulting in a protrusion with symmetric or asymmetric overgrowth (Golladay ES., 2003).

Nowadays, several authors propose nonoperative corrections, such as the body cast or the orthotic compression brace (OCB) (Frey AS, 2006).

Due to the symmetric or asymmetric nature of the deformity, some studies report better outcomes with a custom-fitted OCB (Egan JC, 2000).

Currently, this procedure is performed using rough measurements, which may lead to future adjustments in OCB design; or CT-scan with radiation dosage; or, in few cases, precision 3D scanners which are costly (Philippe, 2007).

In this paper our primary focus is the validation of the Kinect sensor as a handheld scanner for modelling Pectus Carinatum OCB. To this extent, it is compared and discussed the differences between the 3D scans and errors that affect the OCB modelling from a software using Kinect (ReconstructMe), the mesh from an available handheld scanner (Polhemus FastSCAN) and the mesh from the CT-Scan as reference.

The paper proceeds with the description of the acquisition procedure and reliability assessment for a phantom model, and also the measurements for the OCB modelling. In Section 3, the errors obtained from different scans and OCB measurements are presented. The paper concludes with some observations and future improvements in Section 4.

\section{METHODS}

\subsection{D Scanning}

\subsubsection{FastSCAN}

The Polhemus FastSCANTM Cobra is a handheld scanner which uses a camera and a laser to triangulate a 3D stripe. An electromagnetic tracking system is used to track the scanner wand location in the 3D space.

Resolution along the laser line depends on wandobject range, typically $0.5 \mathrm{~mm}$ at $200 \mathrm{~mm}$ range and it can reach resolutions as low as $0.1 \mathrm{~mm}$. The distance between transmitter (small ranger) and wand is limited to a radius of $310 \mathrm{~mm}$, so a good accuracy is 
achieved $(0.75 \mathrm{~mm})$ within a $600 \mathrm{~mm}$ sphere centered on the reference source (Polhemus, 2012).

\subsubsection{D Kinect (ReconstructMe Software)}

Kinect is a device composed by one Infra-Red (IR) projector, one IR camera and one RGB camera. The IR projector and IR camera are used to triangulate the points in space, and to estimate the depth by measuring the disparities captured by the IR camera (Smisek, 2011, Khoshelham, 2012).

The operating range of the sensor is between 0.4 meters to 5 meters. At the range of 2 meters, one level of disparity corresponds to $1 \mathrm{~cm}$. Thus, to increase the depth resolution for acquisitions with Kinect, the acquisition range is limited to 0.4 meters up to 1.2 meters. According to Khoshelham et. al., the standard deviations of depth resolution at 1.5 meters can be as high as $0.5 \mathrm{~cm}$.

The software ReconstructMe (Non-commercial version 405), developed by PROFACTOR GmbH, was used to build the surface meshes. (ReconstructMe, 2012). Essentially, ReconstructMe uses depth acquisitions to represent 3D points, which characterize a 3D scene.

\subsection{Reliability assessment}

In order to access and validate the differences between scanners capability to scan the human chest wall, a phantom (Training Model "ABDFAN" Kyoto Kagaku Co., Ltd) was used in this analysis. The usage of Kinect is then evaluated for OCB measures by assessing its similarity with FastSCAN and CT-Scan results.

The surface mesh reconstructed from the CTdata is used as the ground-truth in this study. The volume resolution is $512 \times 512 \times 241$ with voxel dimensions of $0.684 \times 0.684 \times 1 \mathrm{~mm}$, the 241 axial slices were acquired with the HiSpeed CT/e $\mathrm{e}^{\mathrm{TM}}$ (GE Medical Systems).

The surface contours from the segmented slices are used to reconstruct the final mesh, see Figure 1.

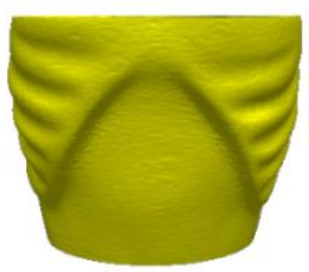

Figure 1: Surface mesh from CT-scan.

Two different setups were performed:
- Movement - the scanner moves around a static object;

- Static - the scanner is fixed and the object moves in front of it.

To improve the static mode, the object is fixed in a support which allows 360 degrees rotation. For each mode and scanner, 10 meshes were acquired.

\subsubsection{Repeatability}

The FastSCAN scanner is operator dependent, since the mesh precision depends of the distance between the wand and the reference. Occlusion is another problem which brings the necessity of extra sweeps. Therefore, to overcome these limitations, some post-processing was applied to the meshes. First, the sweeps were slightly registered to decrease the distances between them. Then, smooth and decimate operators were applied to the merged meshes

The repeatability was also studied in Kinect based on ReconstructMe software with default settings.

To analyse the repeatability, the CloudCompare software running the ICP algorithm was used to align the meshes. Two hundred thousand sampling points were used. Then, the registration was validated by Root-Mean-Square (RMS) error between meshes. The distance between meshes was computed assigning each point of the compared mesh to the nearest-neighbour point in the reference mesh.

Four different setups were defined and, for each, 10 meshes were acquired. To compute the repeatability, the described process was applied to all meshes. To reduce the influence of the registration in error measurements, due to different number of vertexes per mesh, the comparisons were made through the combination of all meshes, using all of them as reference. In each setup 90 comparisons were computed.

\subsubsection{Accuracy}

This subsection describes how the meshes accuracy was accessed. Accuracy represents the distance between the corresponding points of the surface mesh acquired from the scanner and the ground-truth surface mesh.

Here, they were applied the same steps of the repeatability, however, for this case, the acquired meshes were compared with ground-truth mesh built from the CT-scan. The accuracy is measured and 
compared in the four setups, resulting in a total of 40 comparisons.

\subsection{OCB modelling}

Usually, the OCB is modelled by taking measurements from CT-Scan or, if there is no available patient CT-Scan, measured manually in the patient. In the CT-data, one slice is chosen at the point of greatest protrusion. The measurements for modelling the OCB are the transverse diameter of the thorax (Figure $2-\mathrm{A}$ ), the right and left hemithorax distance (Figure $2-\mathrm{B}$ and $\mathrm{C}$ ) and the thorax perimeter. The curvature of the anterior and posterior elements of the OCB (Figure $2-D_{a}$ and $D_{p}$ ) are modelled following the lateral tangential curvature of the chest.

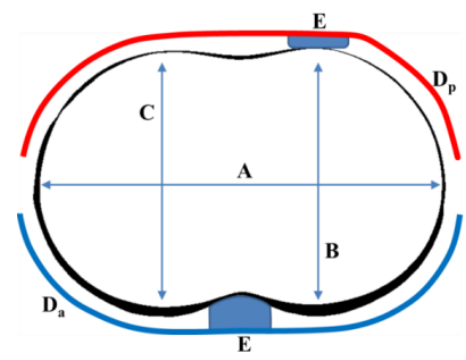

Figure 2: Measurements for modelling the OCB: A transverse diameter of the thorax; $\mathrm{B}$ and $\mathrm{C}-$ anteroposterior distance of the right and left hemithorax. $D_{a}$ and $D_{p}$ - anterior and posterior elements of the OCB, E - Contact pillow/support.

To realise if the OCB modelling can be achieved using 3D scanners, the defined measurements were computed in both scan meshes and compare to the CT-Scan mesh in the greatest protrusion point.

\section{RESULTS AND DISCUSSION}

For each scan preformed with the Kinect, the phantom was turned around $360^{\circ}$, at least 3 times, to minimize random noise.

When the mean and standard deviation were computed, the outliers were eliminated using $99.73 \%(3 \sigma)$ of the total data for each mesh comparison.

\subsection{Repeatability}

The results reveal that the Kinect acquisition based on ReconstructMe has higher errors for both modes. The RMS errors, obtained from registration, are reported in Figure 3.

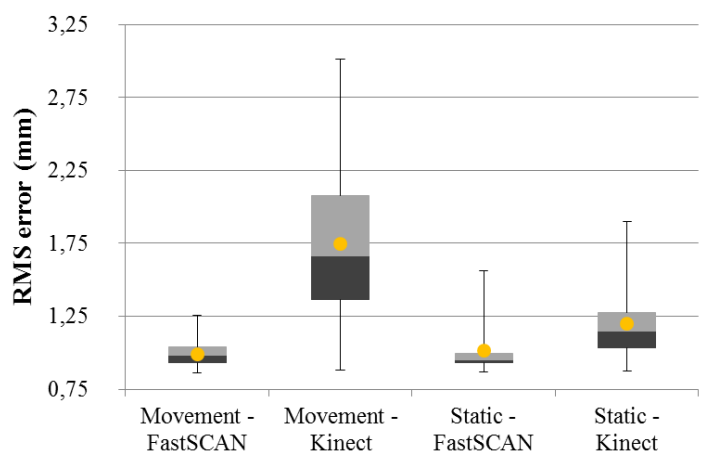

Figure 3: Repeatability - Boxplot results of the RMS error for the four setups. Mean value is represented by the yellow dot.

As the differences are not substantial, it is possible to mention that FastSCAN performs repeatable acquisitions in both modes. Using Kinect, the results reveal that repeatability is higher when the sensor stays static and the object moves. When the Kinect is used as a handheld scanner (movement mode), the influence of the operator is verified - on average higher than $0.5 \mathrm{~mm}$.

\subsection{Accuracy}

The accuracy results are presented in Figure 4, revealing that FastSCAN is more accurate than Kinect.

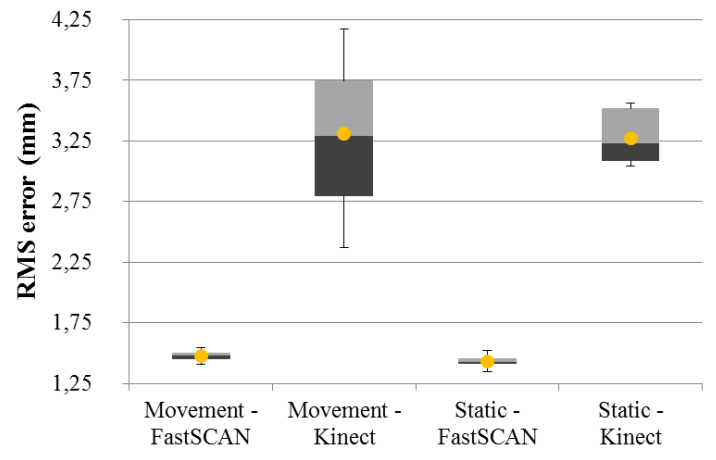

Figure 4: Accuracy - Boxplot results of the RMS error for the four setups for. Mean value is represented by the yellow dot.

On average, the accuracy differences between scanners are higher than $1.8 \mathrm{~mm}$ in RMS error. Although, when comparing acquisition modes 
(movement and static), these do not greatly influence the resulting meshes in either scanners.

\subsection{Corrective bar similarity}

Thorax perimeter, transverse diameter, left and right hemithorax distances are reported in table 1. This table describes the mean values of the 10 meshes of each setup.

Table 1: OCB thorax mean distances, in millimetres.

\begin{tabular}{|l|r|r|r|r|r|}
\hline Setup & TP & TTD & $\begin{array}{r}\text { Left } \\
\text { HTD }\end{array}$ & $\begin{array}{c}\text { Right } \\
\text { HTD }\end{array}$ & $\%$ \\
\hline CT (Ref) & 858,02 & 338,38 & 218,06 & 216,69 & \\
\hline Kinect & 842,30 & 332,29 & 211,16 & 211,03 & 97,65 \\
(MM) & $\pm 6,28$ & $\pm 1,72$ & $\pm 1,49$ & $\pm 3,87$ & $\pm 0,93$ \\
\hline Kinect & 855,60 & 328,38 & 206,00 & 216,10 & 97,74 \\
(SM) & $\pm 7,07^{\Delta}$ & $\pm 0,96$ & $\pm 1,74$ & $\pm 0,85^{\Delta}$ & $\pm 0,57$ \\
\hline FastSCAN & 865,85 & 339,77 & 217,93 & 218,88 & 100,57 \\
(MM) & $\pm 2,78$ & $\pm 0,49^{\Delta}$ & $\pm 1,02^{\Delta}$ & $\pm 0,54^{\square}$ & $\pm 0,30$ \\
\hline FastSCAN & 853,35 & 334,45 & 215,63 & 213,21 & 98,89 \\
(SM) & $\pm 3,54^{\square}$ & $\pm 0,71^{\square}$ & $\pm 0,95^{\square}$ & $\pm 0,61$ & $\pm 0,33$ \\
\hline
\end{tabular}

TP - thorax perimeter; TTD - thorax transverse distance; HTD - Hemithorax distance; $\%$ - percentage of similarity with CT-data; SM - static mode; MM - movement mode. $\Delta$ - best result; $\square-$ second best result.

The best performance was achieved using the FastSCAN in MM setup (see Table 1 - \%). Also, the overall best results are achieved with FastSCAN scanner in both modes, MM and SM.

Observing the difference of similarity in Kinect $(\sim 2.5 \%)$, this can result in mean error between $5 \mathrm{~mm}$ and $16 \mathrm{~mm}$, affecting the OCB modelling. In the FastSCAN case, with difference of similarity lower than $1.2 \%$, the worst mean error is $7.8 \mathrm{~mm}$.

\section{CONCLUSIONS}

FastSCAN has revealed to be the most accurate and precise scanner. Kinect, with ReconstructMe software, has proved to be a well capable system for the acquisition of 3D objects, demonstrating a RMS accuracy error up to $3 \mathrm{~mm}$, higher than FastSCAN $(\sim 1.5 \mathrm{~mm})$, when compared to ground-truth. Also, it shows less level of detail than FastSCAN.

Since Kinect is a static acquisition system, it shows more variability when used as a handheld. Unlike it, FastSCAN remains stable in both motion setups, SM and MM.

One major drawback of FastSCAN system is its cost when compared to Kinect.
Future improvements in Kinect registration and depth field sensor can expand the usage of this scanner as a low-cost handheld device allowing for fast and precise remote scans for custom-fitted OCB modelling.

\section{ACKNOWLEDGEMENTS}

The authors acknowledge to Foundation for Science and Technology (FCT) - Portugal for the fellowships with the references: UMINHO/BI/95/2012; $\quad$ SFRH/BD/68270/2010; SFRH/BD/74276/2010 and SFRH/BPD/46851/2008. This work was also supported by FCT R\&D project PTDC/SAU-BEB/103368/2008.

\section{REFERENCES}

Golladay, E.S., 2003. Pectus carinatum and other deformities of the chestwall. In Ziegler, M.M., Azizkhan, R.G., Weber, T.R. Operative pediatric surgery. New York (NY) McGraw-Hill, p. 269-77.

Frey, A.S., Garcia, V.F., Brown, R,L, Inge, T.H., Ryckman, F.C., Cohen, A.P., Durrett, G., Azizkhan, R.G., 2006. Nonoperative management of pectus carinatum. In J Pediatr Surg, 41, p. 40-5.

Egan, J.C., DuBois, J.J., Morphy, M., et al., 2000. Compressive orthotics in the treatment of asymmetric pectus carinatum: a preliminary report with an objective radiographic marker. In J Pediatr Surg, 35, p. 1183-6.

Philippe Poncet, Dragan Kravarusic, Tessa Richart, Rhiannon Evison, Janet L. Ronsky, Ali Alassiri, David Sigalet, Clinical impact of optical imaging with 3-D reconstruction of torso topography in common anterior chest wall anomalies, Journal of Pediatric Surgery, Volume 42, Issue 5, May 2007, 898-903.

Smisek, J., Jancosek, M., Pajdla T., 2011. 3D with Kinect. In IEEE International Conference on Computer Vision Workshops, p.1154-60

Khoshelham, K., Elberink, S.O., 2012. Accuracy and Resolution of Kinect Depth Data for Indoor Mapping Applications. In Sensors, 12, p. 1437-54.

Polhemus, 2012. http://www.polhemus.com/ (accessed August 2012).

ReconstructMe, 2012. http://reconstructme.net/ (accessed August 2012) 